\title{
Case Report \\ Paraurethral Leiomyoma in a Postmenopausal Woman: First European Case
}

\author{
Susy Shim, ${ }^{1}$ Camilla Skovvang Borg, ${ }^{2}$ Huda Galib Majeed, ${ }^{2}$ and Peter Humaidan ${ }^{3,4}$ \\ ${ }^{1}$ Department of Gynecology and Obstetrics, Aalborg University Hospital, Reberbansgade 15, 9000 Aalborg, Denmark \\ ${ }^{2}$ Department of Gynecology and Obstetrics, Viborg Regional Hospital, Heibergs Allé 4, 8800 Viborg, Denmark \\ ${ }^{3}$ The Fertility Clinic, Skive Regional Hospital, Resenvej 25, 7800 Skive, Denmark \\ ${ }^{4}$ Faculty of Health, Aarhus University, Nordre Ringgade 1, 8000 Aarhus, Denmark
}

Correspondence should be addressed to Susy Shim; susyshim@gmail.com

Received 30 January 2015; Accepted 15 March 2015

Academic Editor: Yoshio Yoshida

Copyright (C) 2015 Susy Shim et al. This is an open access article distributed under the Creative Commons Attribution License, which permits unrestricted use, distribution, and reproduction in any medium, provided the original work is properly cited.

Leiomyomas are benign tumors extending from smooth muscle cells and only few cases of paraurethral leiomyomas have been described in the literature. They are often seen in the reproductive age and around $50 \%$ of the cases are asymptomatic. We describe a 59-year-old woman with a solid mobile tumor below the symphysis revealed at a gynecological examination. Transvaginal ultrasound and MRI confirmed the tumor and excision of the paraurethral tumor was carried out. The histological examination showed a benign paraurethral leiomyoma. The postoperative period was characterized by urethral pain as well as vaginal leakage of urine.

\section{Introduction}

Leiomyomas are benign tumors extending from smooth muscle cells. They are the most common type of tumors in the female reproductive organs, but they are rare outside the uterus. Only few cases of paraurethral leiomyomas (PL) have been described in the literature. They are often seen in the reproductive age and around $50 \%$ of the cases are asymptomatic.

We hereby present the first European case of a paraurethral leiomyoma in a postmenopausal woman.

\section{Case}

A 59-year-old woman was referred to a gynecological outpatient clinic suspected to have an ovarian cyst. Some months earlier she had been examined at the general surgery department due to abdominal pain.

The patient had previously undergone a hysterectomy and was medically treated for diabetes. She was diagnosed with diverticulosis and the suspicion of a cyst in the left ovary. She had experienced constipation problems and pain in the lower left side of the abdomen. The pain increased during movement and when eating. The constipation was improved by lactulose treatment, and as a result she only suffered from abdominal pain during physical activity. Importantly, she also reported occasional incontinence, alternating with difficulties emptying the bladder.

A gynecological examination revealed a solid mobile tumor below the symphysis, measuring $3 \times 3 \mathrm{~cm}$. A transvaginal ultrasound examination showed two normal ovaries and normal findings in the lower pelvis. In contrast, a tumor measuring $2.8 \times 2.3 \mathrm{~cm}$ closely related to and intruding into the urethral wall was seen.

A magnetic resonance imaging (MRI) revealed a sharply defined tumor, measuring $3 \times 3 \mathrm{~cm}$ closely related to the vaginal vault (Figure 1). The tumor caused an impression of the urethra, but invasive growth was not suspected. A transvaginal biopsy of the process showed smooth muscle tissue without signs of malignancy.

Subsequently, an excision of the paraurethral tumor was carried out in collaboration between urologists and gynecologists. In the beginning of the operation a cystoscopy was performed. A little diverticel was seen in the urethra but there was no observed connection between the paraurethral tumor and the urethra. The operation was performed with only local 


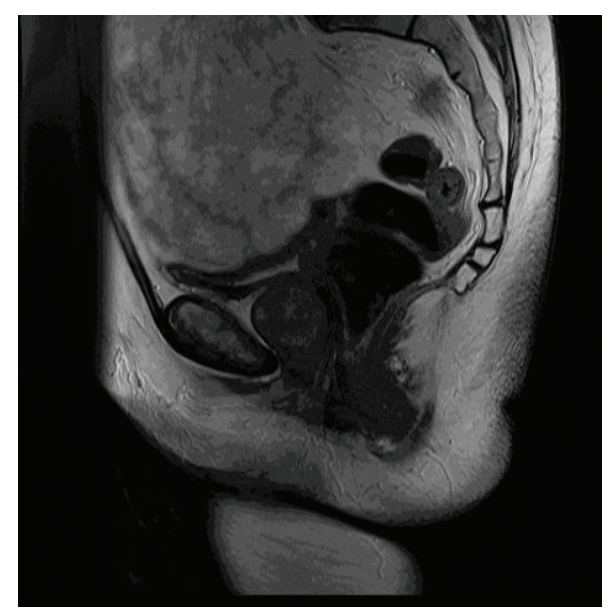

FIGURE 1: MRI of paraurethral leiomyoma in relation to urethra.

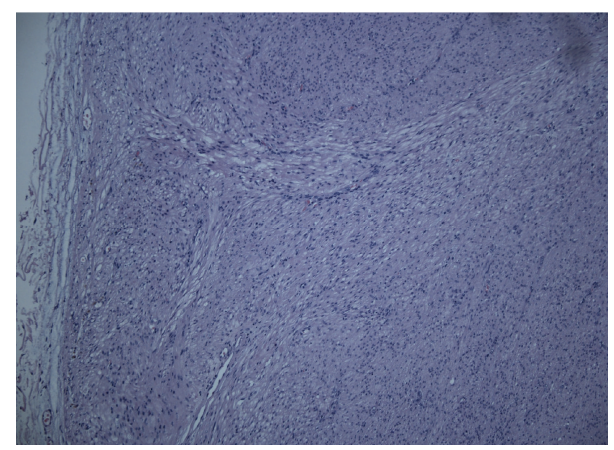

FIGURE 2: Rem of leiomyoma in relation to urethra, HE-stain.

anesthesia. Lidocaine with adrenalin was injected into submucosa in the anterior wall of vagina. The mucosa was divided lengthwise and the tumor was dissected free. During the dissection a minor lesion of the bladder occurred and lesion was sutured in two layers. Injection with methylene blue fluid confirmed no leakage in the lesion. Finally the pubocervical fascia and the mucosa in vagina were sutured. The patient was catheterized for one week and got antibiotic treatment both preoperatively and postoperatively for one week.

The postoperative period was characterized by urethral pain as well as vaginal leakage of urine. A subsequent gynecological examination revealed two vesicovaginal fistulas, which were sutured. Following this the leakage stopped and the patient was free of symptoms.

The histological examination showed a benign leiomyoma (Figure 2).

\section{Discussion}

$\mathrm{PL}$ is rare benign tumors originating from smooth muscle cells $[1,2]$. Leiomyomas occur throughout the genitourinary system, most commonly in the renal capsule [1]. They can occur in both sexes but are predominantly found in women and primarily during the reproductive age [3]. In total less than 30 cases of PL have been documented in Europe since
1977. PL cases are often asymptomatic, but larger tumors might cause symptoms such as pain, voiding problems, and dyspareunia [3]. One case presented with profuse vaginal bleeding [4].

Despite the fact that PLs are benign tumors, most studies recommend that the $\mathrm{PL}$ is removed in order to rule out a leiomyosarcoma [5]. Other differential diagnosis should be made with urethral diverticula, vaginal cysts, urethrocele, Gartner's cysts, and Skene's duct abscess [6].

Without an operation the patient might still experience pressure symptoms with risk of deterioration.

Although a MRI can give an idea of whether a leiomyoma or a leiomyosarcoma should be suspected, the final diagnosis is histological.

Leiomyomas typically appear as round and well-defined structures. There is a low T1 signal intensity and a low to intermediate T2 signal intensity [3]. Degenerated areas, though, may show high intensity. In contrast, leiomyosarcomas are irregular and heterogeneous in structure and with areas of necrosis or hemorrhage $[3,7]$. Furthermore, they have a high level of T2 signal intensity. Findings from the MRI-scan may be compared to findings from the transvaginal biopsy, allowing the surgeon to optimize the operative procedure.

It is believed that ovarian hormones stimulate the development of leiomyomas because they mainly occur in the reproductive age $[2,8]$. It is supported by reports of enlargement during pregnancy and shrinkage after delivery, and it has also been demonstrated in one case with presence of estrogen and progesterone receptors with immunohistological chemical examination $[1,7]$. But some authors have suggested that ovarian hormones do not influence the development because, as in our case, the tumor also occurs in a postmenopausal woman. So further studies with larger population are needed to make any conclusions.

In our case neither any hormonal studies nor immunohistological chemical examination was performed.

In conclusion, we present the first PL case reported in a postmenopausal European woman. Our case demonstrates the importance of a thorough vaginal exploration, including the entire vagina when uncharacteristic pain occurs in the lower pelvis in combination with voiding problems. even in postmenopausal women.

\section{Conflict of Interests}

The authors declare that there is no conflict of interests regarding the publication of this paper.

\section{Acknowledgment}

The authors would like to thank the Department of Pathology, Viborg Regional Hospital.

\section{References}

[1] H. B. Goldman, S. E. McAchran, and G. T. MacLennan, "Leiomyoma of the urethra and bladder," The Journal of Urology, vol. 177, no. 5, p. 1890, 2007. 
[2] S. Kurokawa, Y. Kojima, K. Tozawa, Y. Hayashi, S. Sasaki, and K. Kohri, "Female paraurethral leiomyoma: immunohistochemical approach to the relationship between leiomyoma and ovarian hormones," The Journal of Urology, vol. 167, no. 3, pp. 1403-1404, 2002.

[3] K. C. Hubert, E. M. Remer, R. R. Rackley, and H. B. Goldman, "Clinical and magnetic resonance imaging characteristics of vaginal and paraurethral leiomyomas: can they be diagnosed before surgery?" BJU International, vol. 105, no. 12, pp. 16861688, 2010.

[4] M. C. Kian, J. Chuang, Y.-L. Tsai, and J.-L. Hwang, "A rapidly growing paraurethral myoma with profuse bleeding from a mucosal vessel: report of a case," Gynecologic and Obstetric Investigation, vol. 61, no. 2, pp. 87-89, 2006.

[5] G. Perugia, M. Ciccariello, F. Pirolli et al., "Paraurethral leiomyoma," Urology, vol. 79, no. 4, pp. e51-e52, 2012.

[6] H. Bruschini, J. C. Truzzi, R. Simonetti et al., "Paraurethral leiomyoma in a female causing urinary obstruction," International Urogynecology Journal and Pelvic Floor Dysfunction, vol. 17, no. 3, pp. 286-289, 2006.

[7] P. Tantbirojn, L. Sansopha, V. Thanakit, and N. Sirisubya, "Paraurethral leiomyoma," Journal of the Medical Association of Thailand, vol. 89, no. 4, pp. 545-549, 2006.

[8] C. L. Shadbolt, F. V. Coakley, A. Qayyum, and S. M. Donat, "MRI of vaginal leiomyomas," Journal of Computer Assisted Tomography, vol. 25, no. 3, pp. 355-357, 2001. 


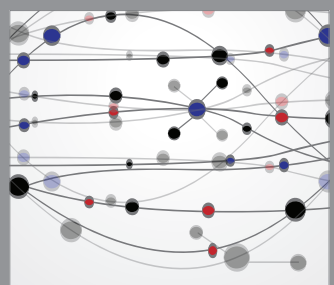

The Scientific World Journal
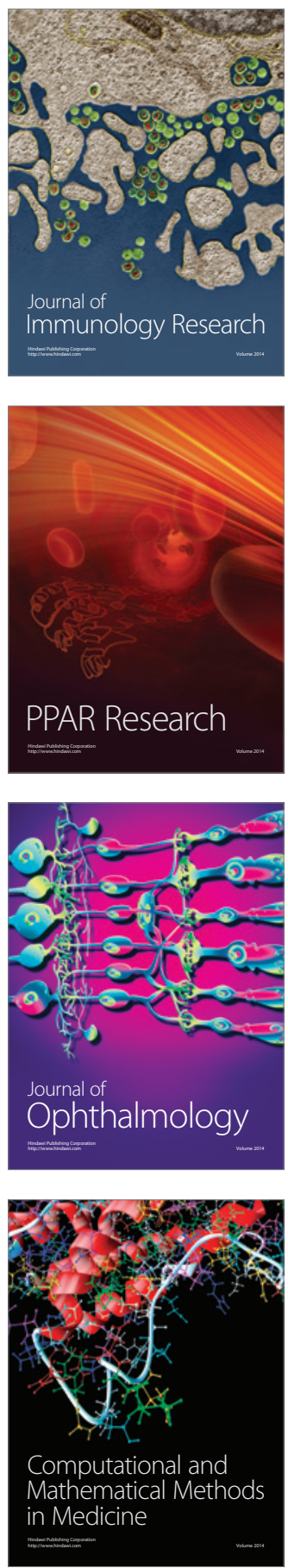

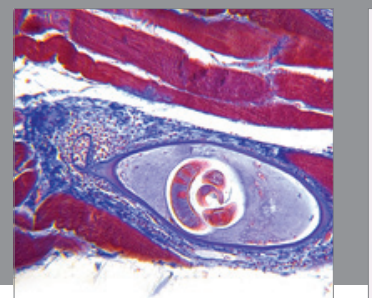

Gastroenterology

Research and Practice
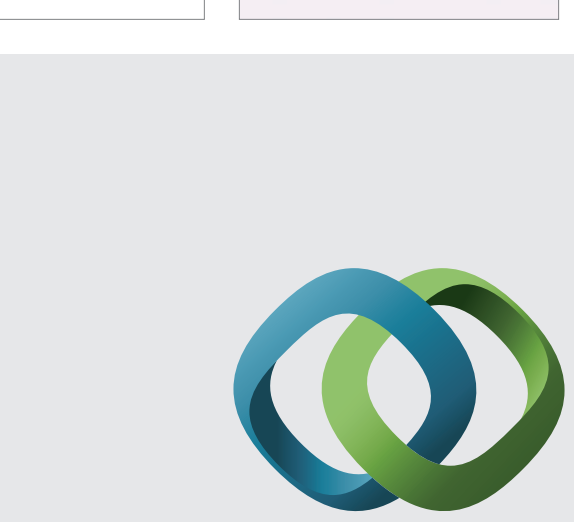

\section{Hindawi}

Submit your manuscripts at

http://www.hindawi.com
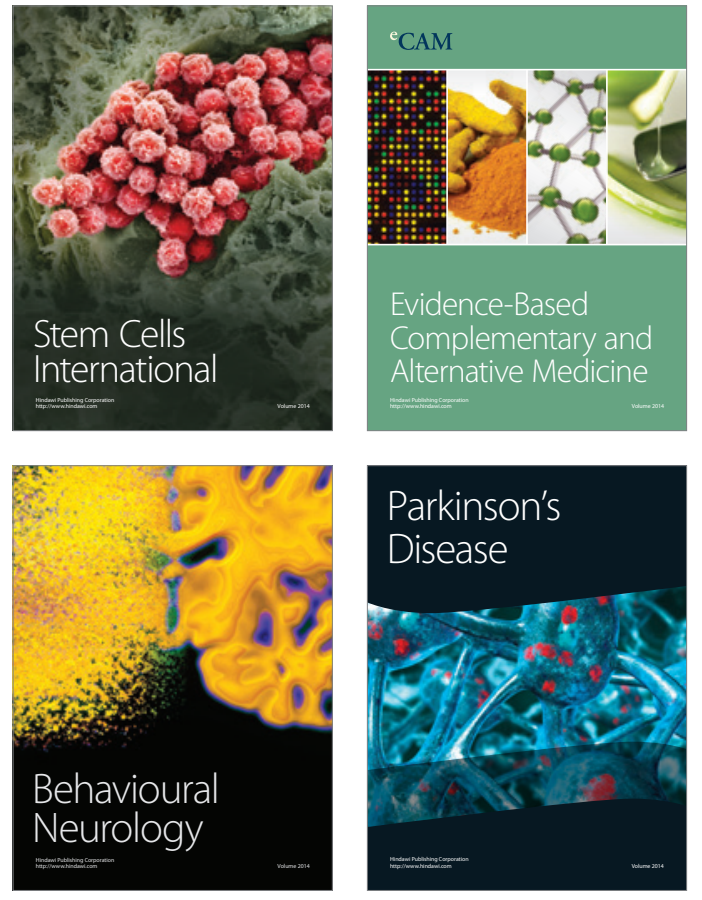
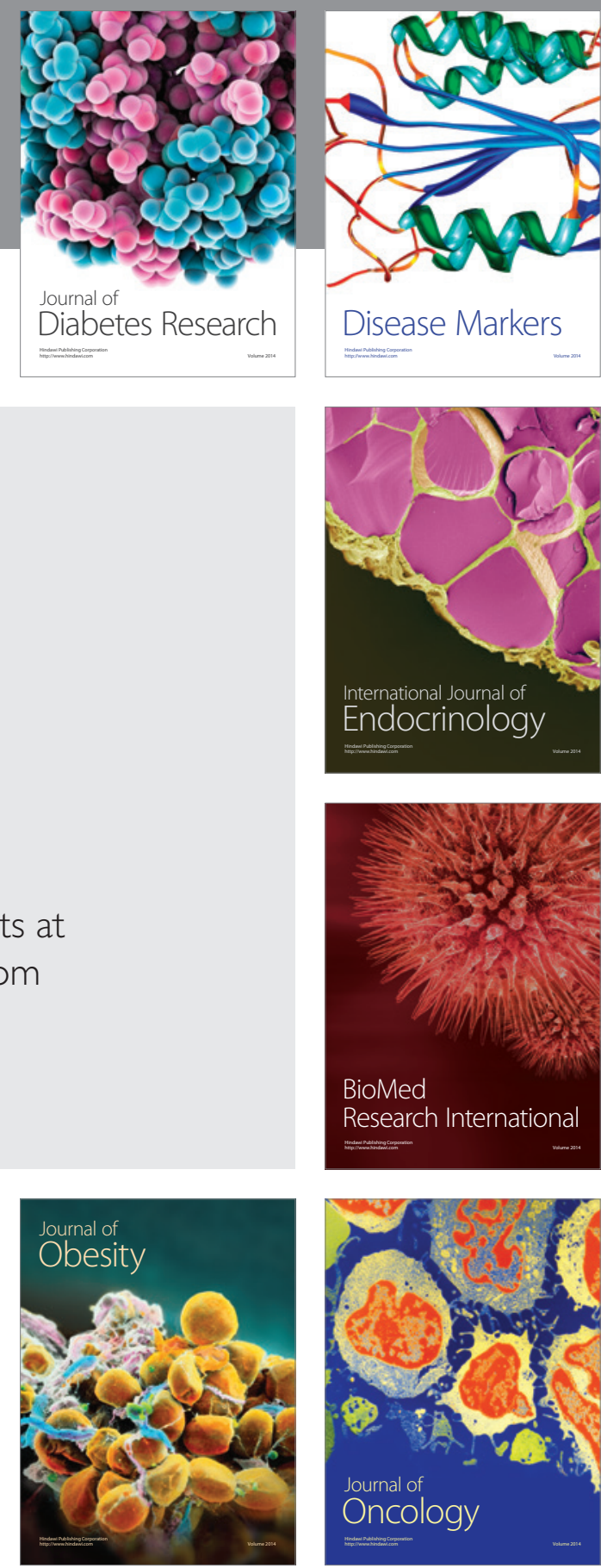

Disease Markers
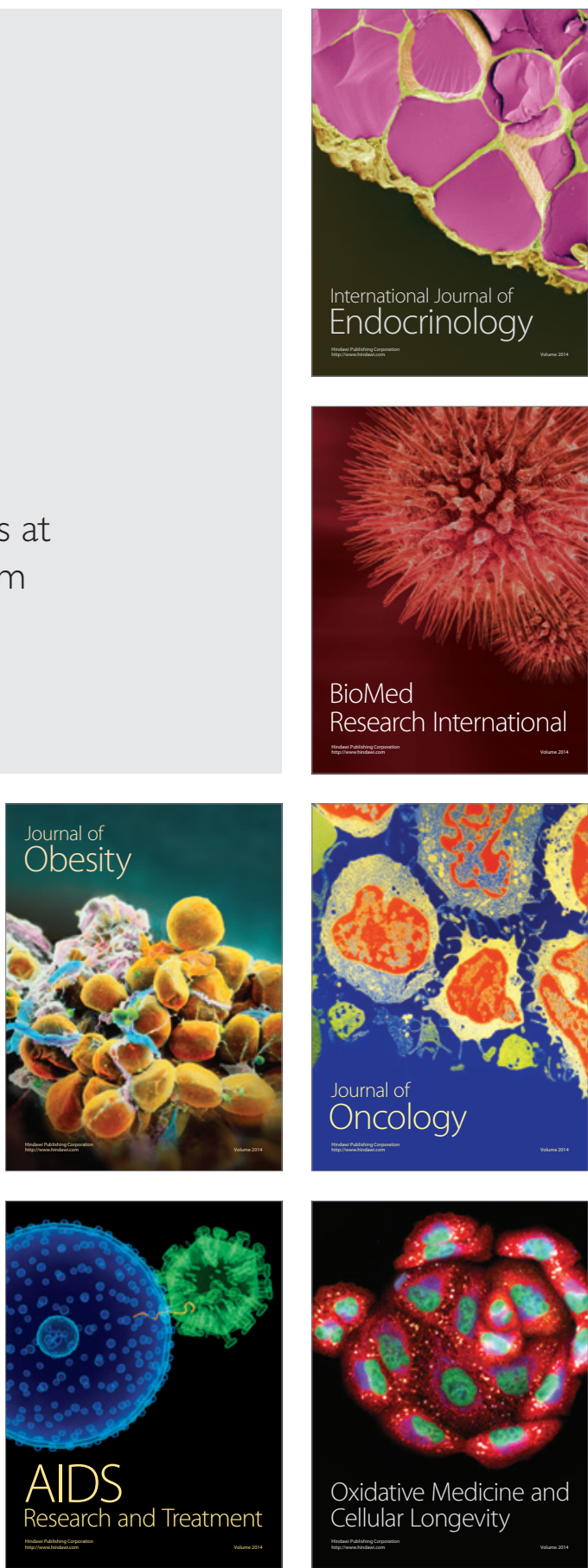